\title{
Preparation and Evaluation of In Vitro Release Kinetics of Theophylline Loaded Matrix Tablet Based on Eudragit NE 30 D and Eudragit RS 30 D
}

\author{
Mohammad Borhan Uddin ${ }^{1}$, J akir Ahmed Chowdhury ${ }^{2}$, Kazi Rashidul Azam ${ }^{3}$, \\ Reza-ul J alil ${ }^{2}$ and Md. Selim Reza ${ }^{2}$ \\ ${ }^{1}$ Department of Pharmacy, North South University, Dhaka, Bangladesh \\ ${ }^{2}$ Department of Pharmaceutical Technology, Faculty of Pharmacy, University of Dhaka, \\ Dhaka-1000, Bangladesh \\ ${ }^{3}$ Department of Pharmacy, Northern University Bangladesh, Dhaka, Bangladesh
}

\begin{abstract}
In the present study efficiency of Eudragit NE 30 D and RS 30 D as matrix forming materials was investigated. It was found that theophylline loaded granules prepared with these two polymers could not sustain drug release for a significant period of time. However, compression of these granules into tablets retarded drug release for up to 7 hours. Release was similar with both of the polymers. Effects of fillers and rate modifiers on drug release have been assessed. Incorporation of lactose and starch caused substantial release of theophylline from both the polymeric systems. Avicel PH 101 intensified the retardation effect of both NE 30 D and RS 30 D on theophylline release. Hydrophobic excipients also show retardation of release from both NE $30 \mathrm{D}$ and RS $30 \mathrm{D}$.
\end{abstract}

Key words: Eudragit RS 30 D, Eudragit NE 30 D, Theophylline, Matrix system, Controlled release

\section{INTRODUCTION}

The fluctuating drug concentrations in blood and tissues caused by conventional dosage forms lead to an insufficient influence on the pathological conditions of diseases and are related to the excessive use of a drug. Various oral dosage forms able to control the rate of drug delivery into the systemic circulation have been prepared and studied. ${ }^{1}$ In spite of the recent technological advances in the fabrication of oral controlled-release dosage forms, particular attention has been paid to the regulation of drug release rate by means of monolithic devices, whereby prior dispersion of the drug in a polymer matrix is carried out. ${ }^{2}$ Embedding a drug within an

Correspondence to: Md. Selim Reza

Tel: +880-2-8612069; Fax: +880-2-8615583

E-mail: selimreza_04@yahoo.com

Dhaka Univ. J. Pharm. Sci. 8(2): 153-159, 2009 (December) insoluble matrix provides a convenient means of controlling the drug release. In such a system, drug release is preceded by penetration of the dissolution medium into the porous matrix to dissolve the drug, followed by diffusion/leaching of the dissolved molecules out of the matrix. Solid drug on the matrix surface will be dissolved and released first. Upon exhaustion of the surface drug, the depletion zone will then increased progressively as the solid drug front recedes into the matrix ${ }^{3}$

A wide array of polymers has been employed as drug-retarding agents each of which presents a different approach to the matrix concept. Plastic matrix systems, due to their chemical inertness and drug embedding ability, have been widely used for sustaining the release of drug. Liquid penetration into the matrix is the rate-limiting step in such systems 
unless channeling agents are used. ${ }^{4}$ Utilization of acrylic polymer as plastic rate-retarding matrix has been reported previously. ${ }^{5}$

In the present study, the comparative potentiality of Eudragit NE 30 D (ethyl acrylate-methyl methacrylate based copolymer in the 2:1 ratio) and Eudragit RS 30 D (poly ethyl acrylate, methyl acrylate, trimrthyl ammonio ethyl methacylate chloride in the 1:2:0.02 ratio) as matrix material for controlling the release of active ingredient has been investigated. Wet granulation process with aqueous dispersion of NE $30 \mathrm{D}$ and RS $30 \mathrm{D}$ was carried out to prepare the controlled-release tablets. Effect of excipients with differential physico-chemical property on theophylline release from RS $30 \mathrm{D}$ and NE 30 D systems have been evaluated.

\section{MATERIALS AND METHODS}

Materials. Materials used in this experiment are theophylline, which was a generous gift from Square Pharmaceuticals Ltd., Bangladesh. Eudragit NE 30 D and Eudragit RS 30 D (Rohm), Avicel PH 101 (FMC Biopolymer, USA), Stearic acid (BDH, UK), carnauba wax (Loba, India), Lactose (The Lactose Co. of New Zealand, New Zealand), starch (Loba
Chemie pvt. Ltd), Aerosil 200 (Degussa, Germany), and Magnesium Stearate (Wilfrid Smith Ltd, UK) were in the study. All the other chemicals used were of analytical grade.

Preparation of wet mass granules. For the preparation of theophylline loaded granules, $10 \mathrm{ml}$ of polymer dispersion (Eudragit NE 30 D/ Eudragit RS $30 \mathrm{D})$ was added part by part to $20 \mathrm{~g}$ of theophylline with continuous blending. The wet mass was passed through 10 mesh sieve followed by 20 mesh. Finally the granules were passed through $1 \mathrm{~mm}$ sieve. These granules were subjected to dissolution studies.

Preparation of compressed tablets. For each batch of matrix formulation $300 \mathrm{mg}$ of prepared granules were measured in an electronic balance and mix with previously measured $100 \mathrm{mg}$ of fillers/release modifier in a blender. A mixing time of 15 minutes was used to produce homogenous granules-excipient mixture. The formulation code of tablets and granules are presented in Table 1. The appropriate amount of mixture were then compressed using a Perkin-Elmer laboratory hydraulic press equipped with $13 \mathrm{~mm}$ flat faced punch and die set. The compression force and compression time were 5 ton and 30 seconds respectively.

Table 1. Composition of different formulations (mg) of matrix tablets

\begin{tabular}{lcccccccc}
\hline & Theophylline & $\begin{array}{c}\text { Eudragit } \\
\text { NE 30 D }\end{array}$ & $\begin{array}{c}\text { Eudragit } \\
\text { RS 30 D }\end{array}$ & Starch & Lactose & $\begin{array}{c}\text { Avicel } \\
\text { PH101 }\end{array}$ & $\begin{array}{c}\text { Carnauba } \\
\text { wax }\end{array}$ & $\begin{array}{c}\text { Stearyl } \\
\text { alcohol }\end{array}$ \\
\hline NE-S & 260 & 40 & - & 100 & - & - & - & 260 \\
NE-L & 260 & 40 & - & - & 100 & - & - & - \\
NE-A101 & 260 & 40 & - & - & - & 100 & - & 40 \\
NE-CAR & 260 & 40 & - & - & - & - & 100 & - \\
NE-SA & 260 & 40 & - & - & - & - & - & 100 \\
RS-S & 260 & - & 40 & 100 & - & - & - & - \\
RS-L & 260 & - & 40 & - & 100 & - & - & - \\
RS-A101 & 260 & - & 40 & - & - & 100 & - & - \\
RS-CAR & 260 & - & 40 & - & - & - & 100 & - \\
RS-SA & 260 & - & 40 & - & - & - & - & 100 \\
\hline
\end{tabular}

Dissolution studies. In vitro drug release studies from the prepared matrix tablets were conducted using a six stations USP XXII type 1 apparatus at $37 \pm 0.5^{\circ} \mathrm{C}$ and $50 \mathrm{rpm}$ speed. The dissolution studies were carried out in duplicate for 8 hours in distilled water under sink condition. At specific time interval samples of $10 \mathrm{ml}$ were withdrawn from the dissolution medium and replaced with fresh medium to maintain the volume constant. After filtration and appropriate dilution, the sample solution was analyzed at $271 \mathrm{~nm}$ for theophylline by an UV spectrophotometer (Shimadzu, Japan). The 
amounts of theophylline present in the samples were calculated with the help of appropriate calibration curves constructed from reference standard. Drug dissolved at specified time periods was plotted as percent release versus time (hours) curve.

Kinetic modeling of drug release. After completing in vitro dissolution of all the batches for eight hours, the data were treated with zero order equation ${ }^{6}$ and Higuchi equations ${ }^{7}$ (equation 1-2 respectively).

$M_{t}=M_{0}+k_{0} t \ldots \ldots \ldots \ldots \ldots \ldots \ldots \ldots$ (1)

$M_{t}=M_{0}-k_{H} t^{1 / 2}$

In these equations, $M_{t}$ is the cumulative amount of drug released at any specified time ( $t$ ) and $\mathrm{M}_{0}$ is the dose of the drug incorporated in the delivery system. $\mathrm{k}_{0}$ and $\mathrm{k}_{\mathrm{H}}$ are rate constants for zero order and Higuchi model respectively. These models failed to explain drug release mechanism due to swelling (upon hydration) along with gradual erosion of the matrix. Therefore the dissolution data were also fitted to well-known Korsmeyer kinetic equation ${ }^{8}$ to ascertain the mechanism of drug release.

$\log \left(M_{t} / M_{\infty}\right)=\log k+n \log t$.

Where $\mathrm{M}_{\infty}$ is the amount of drug release after infinite time; $\mathrm{k}$ is the release rate constant which considers structural and geometric characteristics of the tablet; and $\mathrm{n}$ is the diffusional exponent or release exponent; indicative of the mechanism of drug release. For a tablet having cylindrical shape, when $\mathrm{n}$ is below 0.45, the Fickian diffusion phenomenon dominates, and $\mathrm{n}$ between 0.45 and 0.89 is an anomalous transport (non-Fickian diffusion), often termed as first-order release. After the $\mathrm{n}$ value reaches 0.89 and above, the release can be characterized by case II and super case II transport, which means the drug release rate does not change over time and the release is characterized by zero order. In this case, the drug release is dominated by the erosion and swelling of the polymer. ${ }^{9-10}$

Statistical analysis. The data was subjected to ANOVA for analyzing the statistical difference.

\section{RESULTS AND DISCUSSION}

Theophylline granules were prepared with two different polymers, Eudragit NE 30 D and Eudragit RS 30 D. Compressed tablets of the granules were also prepared with different types of release modifier such as Starch, Lactose, Avicel PH 101 , Carnauba wax and Stearyl alcohol. Both granules and compressed tablet were then evaluated for in vitro dissolution study in distilled water.

The tablets of different formulations were subjected to various evaluation tests, such as weight variation, friability, hardness, and content uniformity according to procedure specified in Indian Pharmacopoeia. The weight variation and friability was less than $6 \%$ and $0.5 \%$, respectively. Good uniformity in drug content was found among different batches of the tablets, and the drug content was more than $95 \%$ (Table 2). A linear relationship was observed between the hardness and the disintegration time (Figure 1). Maximum disintegration time was found 130 minutes in case carnauba wax containing batches of both NE $30 \mathrm{D}$ and S $30 \mathrm{D}$ where the hardness values were also found maximum as $11.1 \pm 0.2$ and $10.2 \pm 0.3$ respectively.

Table 2. Characterization of matrix tablet of Theophylline*

\begin{tabular}{|c|c|c|c|c|c|c|c|c|c|c|}
\hline Parameter & NE-S & NE-L & NE-A 101 & NE-CAR & NE-SA & RS-S & RS-L & RS-A 101 & RS-CAR & RS-SA \\
\hline Weight variation & $401 \pm 4.0$ & $402 \pm 2.0$ & $399 \pm 3.0$ & $405 \pm 2.0$ & $402 \pm 3.0$ & $403 \pm 4.0$ & $402 \pm 2.0$ & $401 \pm 5.0$ & $401 \pm 2.0$ & $402 \pm 4.0$ \\
\hline Friability (\%) & 0.33 & 0.42 & 0.18 & 0.17 & 0.23 & 0.44 & 0.36 & 0.31 & 0.17 & 0.33 \\
\hline Hardness $\left(\mathrm{kg} / \mathrm{cm}^{2}\right)$ & $5.4 \pm 0.3$ & $5.7 \pm 0.2$ & $9.4 \pm 0.1$ & $11.1 \pm 0.2$ & $9.3 \pm 0.2$ & $5.6 \pm 0.2$ & $5.8 \pm 0.1$ & $9.1 \pm 0.3$ & $10.2 \pm .3$ & $9.6 \pm 0.2$ \\
\hline $\begin{array}{l}\text { Content } \\
\text { Uniformity (\%) }\end{array}$ & $98.2 \pm 2.1$ & $99.3 \pm 3.3$ & $99.1 \pm 3.3$ & $98.1 \pm 2.4$ & $98.4 \pm 3.5$ & $99.1 \pm 4.1$ & $97.1 \pm 1.1$ & $99.3 \pm 2.2$ & $98.2 \pm 3.1$ & $98.1 \pm 4.4$ \\
\hline $\begin{array}{l}\text { Disintegration } \\
\text { Time (min)) }\end{array}$ & 25 & 15 & 35 & 130 & 125 & 20 & 10 & 30 & 130 & 145 \\
\hline
\end{tabular}




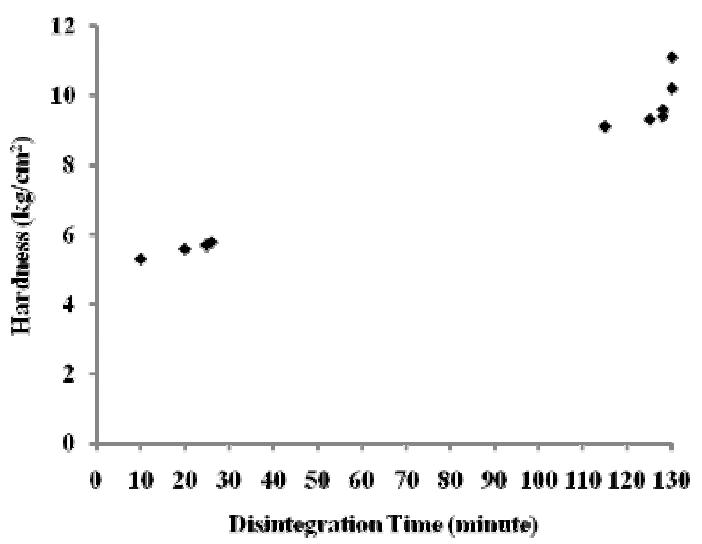

Figure 1. Hardness versus disintegration time relation.

The effects of NE $30 \mathrm{D}$ and RS $30 \mathrm{D}$ polymers on drug release from granules as well as from compressed tablet are presented in Figure 1. Both the polymers could not impart significant sustaining action on drug release from matrix granules $(\mathrm{P}<0.5)$. More than $80 \%$ of theophylline was released from granules prepared with NE 30 D and RS 30 D within 2 hours. Compression of the granules into tablets at 5 -ton compression pressure significantly prolonged the dissolution period for more than 8 hours. Reduction of surface area presented to dissolution fluid is the attributable factor for this observation. Figure 2 also reveals that drug release was almost similar with both of the polymers.

Figure 2 also shows that, after 3 hours $90.21 \%$ of theophylline was released from NE $30 \mathrm{D}$ granules whereas $48 \%$ of theophylline was released from NE $30 \mathrm{D}$ matrix tablet. About $91 \%$ of the active ingredient was released after 3 hour of dissolution from RS 30 D granules whereas $48 \%$ was released from RS 30D matrix tablet.

Eudragit RS $30 \mathrm{D}$ is a copolymer based on ethyl acrylate and methyl methacrylate with plastic characteristics. These polymers are devoid of gel formation and swelling property. The higher rate and extent of theophylline released from NE $30 \mathrm{D}$ is due to greater extraction of theophylline from the vicinity of matrix surface since NE $30 \mathrm{D}$ do not hydrate quickly to form gelatinous surface barrier around the tablet. With the release of surface drug, numerous pores and channels are generated through which dissolution fluid enters into the matrix structure and further elevates the rate and extent of theophylline release.

Figures 3 illustrates the effect of starch, lactose, Avicel PH 101, carnauba wax and stearyl alcohol on release behavior of theophylline from Eudragit NE 30 $\mathrm{D}$ and RS 30 D compressed tablets. Figure $3 \mathrm{a}$ summarizes the effect of the excipients on drug release from NE $30 \mathrm{D}$ polymer based matrix while Figure $3 b$ shows the effect of the excipients on drug

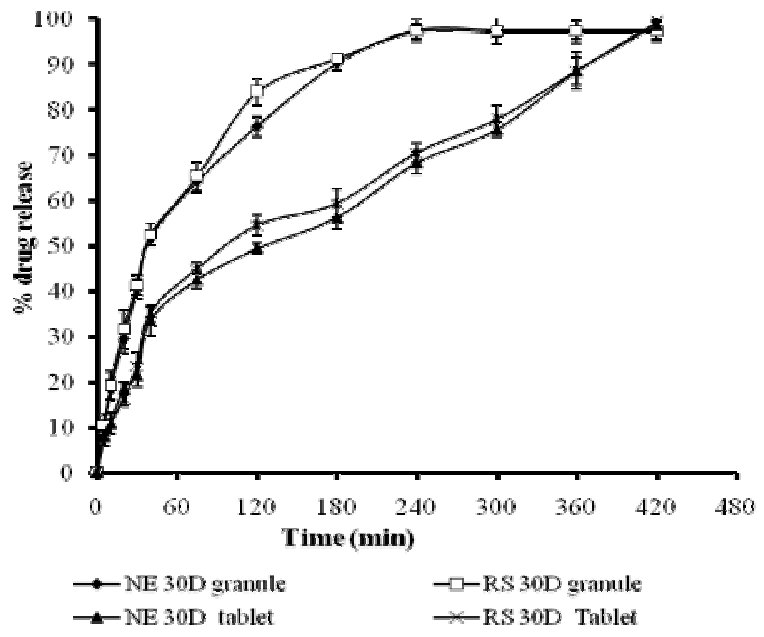

Figure 2. Zero order release profile of theophylline from Eudragit NE 30D and Eudragit RS 30 D. Data are represented as mean \pm $\mathrm{SD}(\mathrm{n}=3)$.

release from RS $30 \mathrm{D}$ polymer based matrix. In case of both of the polymers, incorporation water soluble and water insoluble fillers influenced the release of theophylline significantly from matrix tablet $(\mathrm{P}<$ 0.01). Generally, incorporation of water-soluble excipients results in an increase in the drug release rate due to an increment in total porosity of the matrices due to swelling of starch and lactose (initial porosity plus porosity due to the dissolution of the drug). Lactose is water soluble in nature (1 in 4.63 at room temperature). ${ }^{11}$ Though starch is practically insoluble in cold water, it swells instantaneously in water by about $5-10 \%$ at $35^{\circ} \mathrm{C}^{12-13}$. This is the reason why starch retarded few more drug than lactose. Both carnauba wax and Stearyl alcohol are water insoluble excipients. Carnauba wax is practically insoluble in water and showed good release retarding capacity. Stearyl alcohol is also practically insoluble in water 

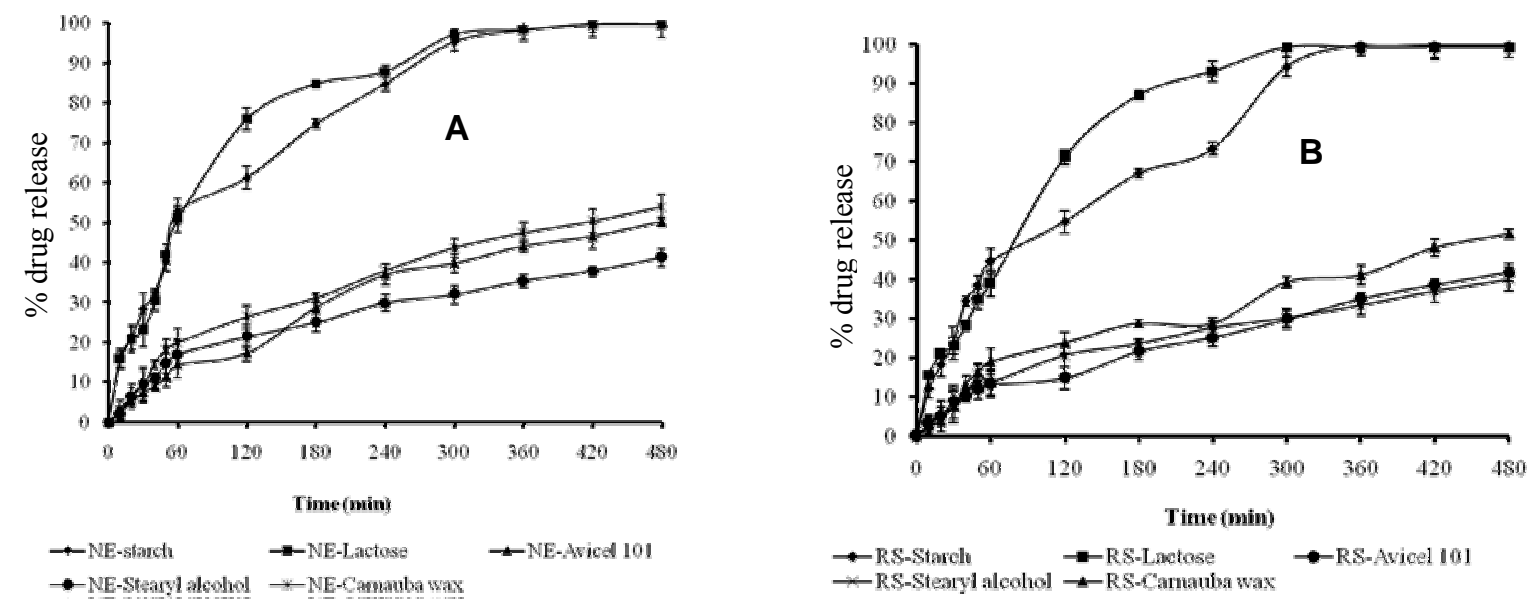

Figure 3. Zero order release profile of theophylline showing effect of excipients on drug release from (a) Eudragit NE 30 D (b) Eudragit RS 30 D. Data are represented as mean $\pm \mathrm{SD}(\mathrm{n}=3)$

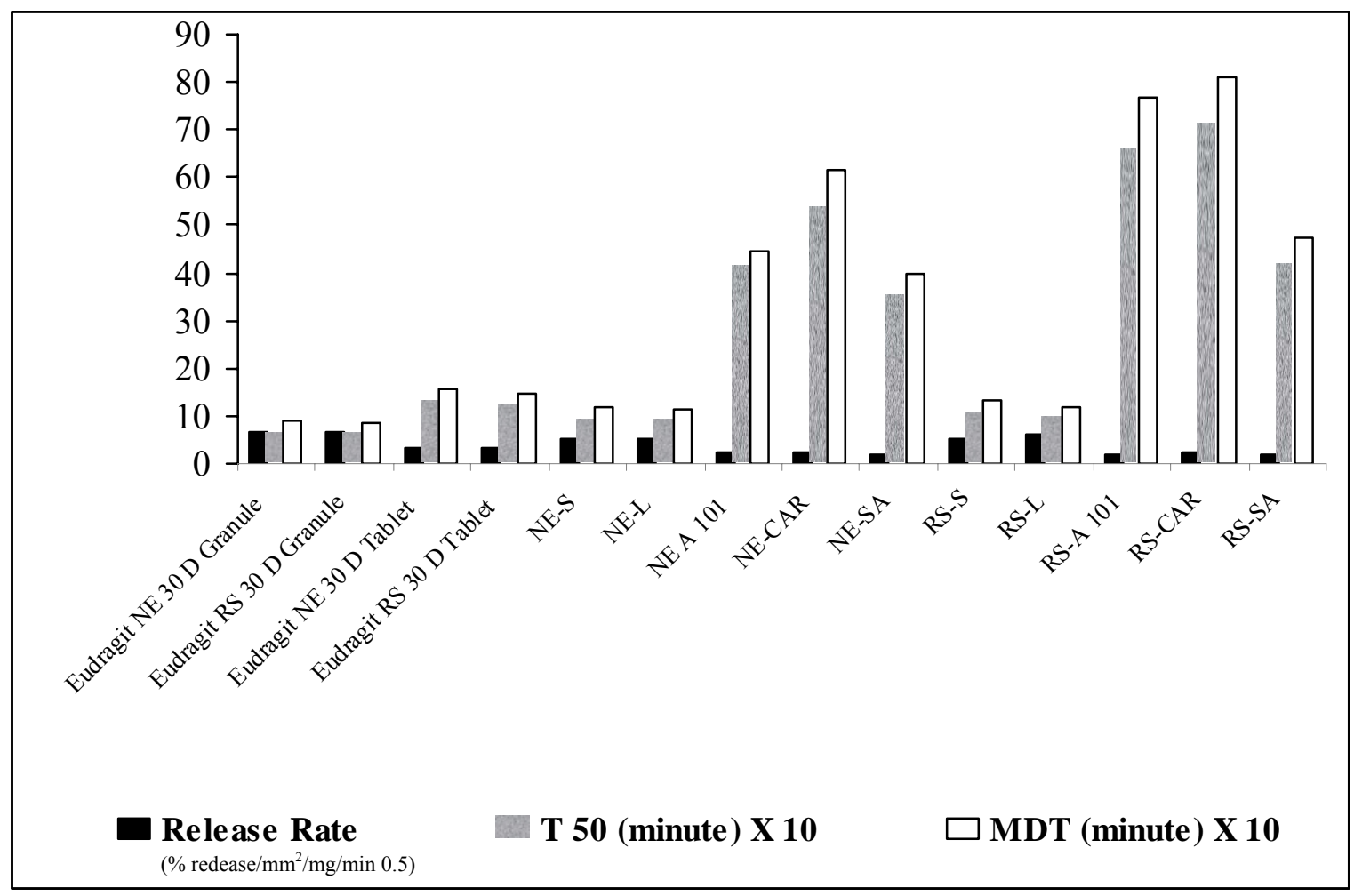

Figure 4. Drug release parameters of different formulas.

and for this reason it has been used in controlled release tablet preparation. ${ }^{14-15}$ Similar controlled release behavior of stearyl alcohol is also seen in case of theophylline matrix tablet in this experiment. But amongst all, Avicel PH 101 released lowest amount of drug from both NE $30 \mathrm{D}$ and RS $30 \mathrm{D}$ matrix systems. Incorporation of Avicel PH 101 which is microcrystalline cellulose retarded drug release. Only $46.5 \%$ of drug was released after 7 hours. This might be due to the water insoluble nature of microcrystalline cellulose. Avicel PH 101 is a highly hydrophilic and gel forming excipient which may have formed gel structure at the pores, reducing hydrodynamic activity at the pores and thereby 
causing delay in the drug leaching out of the matrix tablets. Hydrophobic excipients retard drug release more effectively compared to hydrophilic excipients. It was observed that $37.88 \%$ and $50.4 \%$ theophylline was released after 7 hours from formulation containing carnauba wax and stearyl alcohol respectively. Hydrophobicity of both of these excipients is due to the presence of long chain alkyl group in their structures.

Table 3. Mathematical Modeling and Drug Release Kinetics of Theophylline Conventional and Sustained-Release Matrix Tablets*

\begin{tabular}{lcccccc}
\hline \multirow{2}{*}{ Batch } & \multicolumn{9}{c}{$\mathrm{r}^{2} \dagger$} & $\mathrm{n}$ & Order of Release \\
\cline { 2 - 5 } & Zero Order & First Order & Korsmeyer & Higuchi & & \\
\hline Eudragit NE 30 D granule & 0.761 & 0.879 & 0.939 & 0.926 & 0.491 & Non-Fickian \\
Eudragit RS 30 D granule & 0.734 & 0.888 & 0.931 & 0.909 & 0.481 & Non-Fickian \\
Eudragit NE 30 D tablet & 0.942 & 0.961 & 0.98 & 0.989 & 0.551 & Non-Fickian \\
Eudragit RS 30 D tablet & 0.925 & 0.961 & 0.989 & 0.989 & 0.68 & Non-Fickian \\
NE-S & 0.860 & 0.966 & 0.974 & 0.971 & 0.492 & Non-Fickian \\
NE-L & 0.808 & 0.967 & 0.950 & 0.94 & 0.521 & Non-Fickian \\
NE-A 101 & 0.960 & 0.983 & 0.985 & 0.982 & 0.748 & Non-Fickian \\
NE-CAR & 0.933 & 0.975 & 0.924 & 0.99 & 0.635 & Non-Fickian \\
NE-SA & 0.916 & 0.953 & 0.955 & 0.988 & 0.672 & Non-Fickian \\
RS-S & 0.900 & 0.911 & 0.975 & 0.981 & 0.542 & Non-Fickian \\
RS-L & 0.825 & 0.955 & 0.965 & 0.943 & 0.543 & Non-Fickian \\
RS-A 101 & 0.971 & 0.985 & 0.978 & 0.983 & 0.619 & Non-Fickian \\
RS-CAR & 0.943 & 0.977 & 0.930 & 0.978 & 0.685 & Non-Fickian \\
RS-SA & 0.923 & 0.945 & 0.953 & 0.984 & 0.67 & Non-Fickian \\
\hline
\end{tabular}

NE and RS indicate Eudragit NE 30 D and Eudragit RS 30 D based formulations respectively. S-Starch, L-Lactose, A 101-Avicel PH 101, CAR-Carnauba wax, and S-Stearyl alcohol. *Analyzed by Regression co-efficient method. $\dagger$ Correlation coefficient

Table 4. Dissolution parameters of Theophylline matrix tablet

\begin{tabular}{lcccc}
\hline Batch & $\mathrm{t}_{25 \%}$ (minute) & $\mathrm{t}_{50 \%}$ (minute) & $\mathrm{t}_{75 \%}$ (minute) & MDT* (minute) \\
\hline Eudragit NE 30 D granule & $15.9 \pm 0.1$ & $65.25 \pm 0.2$ & $149.01 \pm 0.4$ & $88.16 \pm 0.5$ \\
Eudragit RS 30 D granule & $14.6 \pm 0.2$ & $61.65 \pm 0.6$ & $143.23 \pm 0.5$ & $84.60 \pm 0.2$ \\
Eudragit NE 30 D tablet & $35.76 \pm 0.1$ & $125.82 \pm 0.2$ & $262.63 \pm 0.1$ & $157.26 \pm 0.7$ \\
Eudragit NE 30 D tablet & $34.34 \pm 0.2$ & $119.47 \pm 0.1$ & $247.73 \pm 0.1$ & $148.51 \pm 0.5$ \\
NE-S & $21.53 \pm 0.4$ & $88.11 \mathrm{v}$ & $200.89 \pm 0.3$ & $118.87 \pm 0.4$ \\
NE-L & $23.25 \pm 0.2$ & $87.95 \pm 0.1$ & $191.54 \pm 0.1$ & $113.96 \pm 0.2$ \\
NE-A 101 & $163.61 \pm 0.4$ & $413.30 \pm 0.2$ & $710.69 \pm 0.2$ & $446.76 \pm 0.3$ \\
NE-CAR & $179.24 \pm 0.5$ & $533.96 \pm 0.4$ & $1011.162 \pm 0.5$ & $617.77 \pm 0.3$ \\
NE-SA & $125.35 \pm 0.2$ & $351.63 \pm 0.1$ & $642.87 \pm 0.1$ & $396.44 \pm 0.2$ \\
RS-S & $29.41 \pm 0.2$ & $105.66 \pm 0.2$ & $223.26 \pm 0.6$ & $133.42 \pm 0.1$ \\
RS-L & $26.40 \pm 0.5$ & $94.62 \pm 0.4$ & $199.66 \pm 0.3$ & $119.34 \pm 0.2$ \\
RS-A 101 & $214.32 \pm 0.1$ & $656.74 \pm 0.5$ & $1264.375 \pm 0.1$ & $769.41 \pm 0.5$ \\
RS-CAR & $248.33 \pm 0.2$ & $712.09 \pm 0.2$ & $1318.72 \pm 0.4$ & $810.34 \pm 0.5$ \\
RS-SA & $148.95 \pm 0.2$ & $419.12 \pm 0.4$ & $767.655 \pm 0.1$ & $473.1514 \pm 0.2$ \\
\hline
\end{tabular}

*MDT indicates mean dissolution time. All values represent mean $\pm \mathrm{SD}(\mathrm{n}=3)$.

Release data were then fitted in different model. Most of the release curves followed Korsmeyer and Higuchi model (Table 3). Non-fickian type release mechanism was observed from all the tablets $(\mathrm{n}<$ 0.85). This means theophylline from matrix tablets of all the formulations were released following diffusion as well erosion ${ }^{9}$. Release rate $(\%$ release
$/ \mathrm{mm}^{2} / \mathrm{mg} / \mathrm{min}^{1 / 2}$ ) of drug were calculated from the slope values of the straight line of the Higuchi plot. The values of release rate of different formulations are shown in Figure 4. Release rate for formulation NE-S, NE-L, NE-A101, NE-CAR, NE-SA were 5.15, $5.38,2.53,2.60$ and 1.99 respectively which shows that release rate is higher with hydrophilic excipinets 
and lower than hydrophobic excipinets. On the other hand release rates of RS-S, RS -L, RS -A101, RS CAR, RS -SA formulations were 5.28, 6.36, 1.91, 2.41 and 1.88 respectively. $\mathrm{T} 50 \%$ and mean dissolution time (MDT) of different formulas were plotted in Figure 4.

Formulations containing Eudragit NE 30 D granules, RS 30 D granules, NE $30 \mathrm{D}$ tablets, RS 30 D tablets have shown better fitting with korsmeyer equation than zero order or first order equation. The $\mathrm{R}^{2}$ values of korsmeyer equation for the above mentioned formulations were $0.9399,0.9316,0.983$, and 0.9826 respectively. Release exponent (n) values of the above mentioned formulation were 0.491, $0.481,0.551$, and 0.681 respectively which indicates that release pattern of the drug was anomalous. The $\mathrm{R}^{2}$ value of korsmeyer equation for formulation NES, NE-L, NE-A101, NE-CAR, NE-SA formulations were $0.9746,0.9505,0.985,0.924$, and 0.955 respectively. The $\mathrm{R}^{2}$ values of first order equation for the above mentioned formulations were 0.9669 , $0.9677,0.9831,0.975$, and 0.9535 respectively. Release exponent (n) values of the above mentioned formulation were $0.492,0.521,0.748,0.635$, and 0.6721 respectively. The $\mathrm{R}^{2}$ value of korsmeyer equation for formulation RS-S, RS -L, RS -A101, RS -CAR, RS -SA formulation were 0.975, 0.9659, $0.978,0.9309$ and 0.953 respectively. Release exponent (n) values of the above mentioned formulation were $0.542,0.543,0.6195,0.685$, and 0.67 respectively.

\section{CONCLUSION}

The experiment reveals that, it is possible to design controlled release drug delivery systems with Eudragit RS 30 D and RS 30 D polymers by wet granulation method. Fabrication of pellets / granules entails the incorporation of stronger rate-retarding agents. Drug release from compressed tablets prepared with NE $30 \mathrm{D}$ and RS $30 \mathrm{D}$ system was found to be critically governed by the excipients present in the system. However, further investigation is required to establish in-vivo-in-vitro correlation to reveal the accurate pattern of drug release in vivo environment from these polymeric systems.

\section{REFERENCES}

1. Bidah, D. and Vergnaud, J.M. 1991. Dosages forms with a polymer matrix and swelling polymer. Int. J. Pharm. 77, 8187.

2. Cardinal, J.R. 1984. Matrix systems. In: RS Langer and D L Wise, editors: Medical application of controlled release, 1st edn., Boca Raton, Florida, CRC Press: 41-67.

3. Billah, N., Yuen, K.H. and Peh, K.K. 1998. Diclofenac release from Eudragit containing matrice and effects of thermal treatment. Drug Dev. Ind. Pharm. 24, 45-50.

4. Reza, M.S., Quadir, M.A. and Haider, S.S. 2003. Comparative evaluation of plastic, hydrophilic and hydrophobic polymers as matrices for controlled release drug delivery. J. Pharm. Pharmaceut. Sci. 6, 282-291

5. Jonvanovic, M., Jovicic G., Duric Z., Agabaga D., Karljikovic K. and Nikolic L. 1997. Effect of fillers and lubricants on Acetylsalicylic acid release kinetics from Eudragit matrix tablet. Drug Dev. Ind. Pharm. 23, 595-602.

6. Mockel, J.E. and Lippold, B.C. 1993. Zero order release from hydrocolloid matrices. Pharm. Res. 10, 1066-1070.

7. Higuchi, T. 1961. Rate of release of medicaments from ointment bases containing drugs in suspension. J. Pharm. Sci. 50, 874-875.

8. Korsmeyer, R.W., Gurny, R., Doelker, E., Buri, P. and Peppas, N.A. 1983. Mechanisms of solute release from porous hydrophilic polymers. Int. J. Pharm. 15, 25-35.

9. Peppas, N.A. 1985. Analysis of Fickian and non-Fickian drug release from polymers. Pharm. Acta. Helv. 60, 110-111.

10. Chueh, H.R., Zia, H. and Rhodes, C.T. 1995. Optimization of sotalol floating and bioadhesive extended release tablet formulations. Drug Dev. Ind. Pharm. 21, 1725-1747.

11. Kibbe A.H. 2000. Hanbook of Pharmaceutical Science, $3^{\text {rd }}$ ed, 279.

12. Ingram J.T. and Lowenthal W.1966. Mechanism of action of starch as a tablet disintegrant I: factors that affect the swelling of starch grains at $37^{\circ} \mathrm{C}$. J Pharm Sci, 55, 614-617.

13. Wurster D.E., Peck G.W. and Kildsig D.O.1982. A comparison of the moisture adsorption-desorption properties of corn starch. USP and ddirectly compressible starch. Drug Dev Ind Pharm, 8, 343-354.

14. Prasad C.M. and Srivastava G.P. 1971. Study of some sustained release granulations of aspirin. Indin $J$ Hosp Pharm, 8, 21-28.

15. Kumar K., Chakrabarti T. and Srivastava G.P. 1975. Studies of the sustained release tablet formulation of diethyl carbamazine citrate (Hetrazan), Indin J Pharm, 37, 57-59. 